\section{Prix Nobel \\ de physiologie \\ ou médecine 2021}

Les senseurs

de la température

et du toucher

Bertrand Coste recherche Scripps à la Jolla (Californie), ont reçu le prix Nobel de physiologie ou médecine 2021 pour «leurs découvertes des récepteurs de la température et du toucher ». Leurs travaux sur la somesthésie, un sens global qui nous renseigne sur les forces physiques s'exerçant au sein de notre organisme et sur notre peau, ont permis de répondre à une question fondamentale: comment les stimulations thermiques et mécaniques sont-elles converties en signaux électriques dans le système nerveux? Ces travaux ont conduit à l'identification des canaux ioniques qui sont à l'interface entre notre corps et son environnement. <

La perception du monde environnant est cruciale pour notre bien-être et notre survie. Cette fonction est assurée par nos sens de la vision, de l'audition, du goût, de l'olfaction et de la somesthésie. La somesthésie est un sens complexe. Ce sens s'articule autour de la détection des molécules chimiques, de la température et des forces mécaniques. Cette détection nous permet, entre autres exemples, de percevoir la température de l'eau, de lire le braille, d'apprécier la dangerosité d'un marteau, et nous renseigne également sur la position de notre corps dans l'espace. Les cellules réceptrices de la somesthésie sont les neurones sensoriels dont les fibres innervent l'ensemble du corps. C'est à l'extrémité de ces fibres, les terminaisons nerveuses situées dans la peau, les muqueuses, les muscles, les articulations et les viscères, que les neurones détectent les stimulus physicochimiques. Un signal est alors généré, puis propagé via différents relais nerveux, aboutissant à la perception consciente, ou non consciente, du stimulus initial. L'initiation du signal électrique s'appelle la transduction sensorielle. Elle est assurée par des canaux ioniques spécialisés qui transforment le stimulus, par exemple

Vignette (๔ Nobel Media. III. Niklas Elmehed).

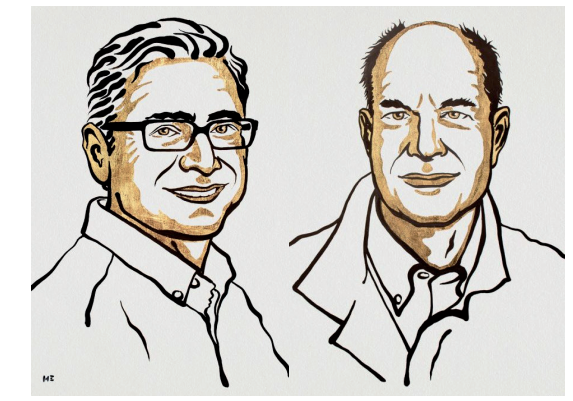

Laboratoire de neurosciences cognitives (LNC)-UMR7291, CNRS/Aix-Marseille Université (AMU),

Faculté de médecine secteur Nord,

13015 Marseille, France.

bertrand.coste@univ-amu.fr

un changement de température ou une contrainte mécanique, en un signal biologique, le passage d'ions de part et d'autre de la membrane des cellules nerveuses qui induit l'excitation de la fibre nerveuse. Les travaux des docteurs David Julius et Ardem Patapoutian sont à I'origine de l'identification de ces senseurs moléculaires.

\section{Les canaux TRP (transient receptor potential) et la thermosensation}

Cette histoire débute dans les années 1990 à San Francisco dans le laboratoire de David Julius (Université de Californie San Francisco, UCSF). David Julius et ses collaborateurs utilisent la capsaïcine, un alcaloïde présent dans les piments rouges qui provoque une sensation de brûlure, afin d'identifier son récepteur. Ils expriment des milliers de gènes, issus de neurones sensoriels, dans un système hétérologue et identifient, par imagerie calcique, celui qui confère aux cellules la sensibilité à la capsaïcine [1], et qui s'avère conférer également la sensibilité à la chaleur. Ce gène code un canal cationique non-sélectif, TRPVI (transient receptor potential cation channel subfamily $V$ member 1). Leur découverte revêt une importance capitale car TRPVI est exprimé spécifiquement par les neurones thermosensibles qui détectent le chaud. II s'active lorsque la température dépasse $42^{\circ} \mathrm{C}$. D'autres études effectuées dan le laboratoire de David Julius montreront, par la suite, que TRPVI est crucial dans la perception douloureuse des températures élevées [2, 3]. 
David Julius est né en 1955 à New York, aux États-Unis. II a obtenu un doctorat en 1984 de l'Université de Californie à Berkeley et a effectué son stage postdoctoral à l'Université Columbia de New York. David Julius a été recruté à l'Université de Californie à San Francisco en 1989 où il est aujourd'hui professeur.

Ardem Patapoutian est né en 1967 à Beyrouth, au Liban. Dans sa jeunesse, il a déménagé d'un Beyrouth déchiré par la guerre à Los Angeles, aux États-Unis, et a obtenu un doctorat en 1996 de l'institut de technologie de Californie (Caltech) à Pasadena. II a effectué son stage postdoctoral à l'université de Californie à San Francisco, et dirige depuis 2000 son laboratoire au Scripps Research Institute à La Jolla, Californie, où il est maintenant professeur.
Le laboratoire de David Julius et celui d'Ardem Patapoutian (The Scripps Research Institute, La Jolla, Californie) vont, par la suite, identifier le senseur du froid, TRPM8 (transient receptor potential cation channel subfamily $M$ [melastatin] member 8), de manière indépendante: David Julius et ses collaborateurs utilisent le menthol, une molécule qui provoque une sensation de frais, dans un crible similaire à celui ayant conduit à identifier TRPVl [4]. Ardem Patapoutian et ses collaborateurs cloneront les gènes présentant une similarité de séquence avec TRPV1, dont fait partie TRPM8 [5]. Ce récepteur est exprimé dans les neurones thermosensibles impliqués dans la détection du froid non douloureux. II s'active pour des températures inférieures à $28^{\circ} \mathrm{C}$, et des souris dépourvues de ce récepteur présentent un déficit majeur de perception du froid [6].

D'autres canaux thermosensibles de cette famille seront ensuite identifiés, notamment TRPAl (transient receptor potential cation channel subfamily $A$ member 1 ), qui est activé par les températures inférieures à $15^{\circ} \mathrm{C}$. TRPAl est également un récepteur de l'ail, du wasabi, une plante d'Asie, et de la moutarde. Il est exprimé dans les neurones nociceptifs responsables de la détection des stimulus douloureux [7]. D'autres études montreront qu'il est activé par une myriade de composés algogènes (qui provoquent une douleur) [8].

La découverte des canaux ioniques thermosensibles a permis de comprendre les mécanismes fondamentaux de la perception de la température. Ces canaux sont également impliqués dans un grand nombre de syndromes douloureux. Les efforts initiaux de l'industrie pharmaceutique pour développer des traitements anti-douleur ciblant ces récepteurs ont été freinés par les nombreux effets secondaires des molécules développées, tels qu'une perception réduite des températures nocives, conduisant à des brûlures chez les patients. Des médicaments de nouvelle génération sont en cours de développement, dont certains ont démontré une efficacité pour traiter des douleurs arthritiques [9].

\section{Les canaux Piezo et la mécanosensation}

La découverte des senseurs du toucher a été une aventure collective à laquelle j'ai contribué. Une décennie après l'identification de TRPVI dans le laboratoire de David Julius, j'ai en effet rejoint le laboratoire
d'Ardem Patapoutian avec pour projet d'identifier les senseurs mécaniques impliqués dans la mécanosensation. Si de tels senseurs étaient caractérisés chez la bactérie et le nématode, les mécanismes moléculaires responsables du toucher chez les vertébrés restaient alors inconnus. En utilisant un dispositif expérimental permettant d'enregistrer directement l'activité électrique induite par la stimulation mécanique des cellules, nous avons pu identifier une lignée cellulaire murine offrant un certain intérêt. Les cellules de cette lignée répondent en effet aux stimulations mécaniques par la genèse de courants électriques similaires à ceux que l'on observe dans les neurones sensoriels. L'analyse des gènes exprimés dans ces cellules a permis de dresser une liste de gènes candidats potentiellement impliqués dans la réponse. Notre stratégie a consisté à bloquer l'expression de ces gènes, un à un, et à tester la réponse de ces cellules aux stimulations mécaniques. Une année de crible minutieux a conduit à l'identification du gène Piezol. PIEZO1, ainsi que PIEZO2, qui sont présents notamment chez la souris et chez l'homme, appartiennent à une nouvelle famille de canaux ioniques activés par les forces mécaniques $[10,11]$. Plusieurs études issues du laboratoire d'Ardem Patapoutian permettront, par la suite, de définir les fonctions de ces gènes dans la somesthésie. Si PIEZOl n'est pas exprimé dans les neurones sensoriels, PIEZO2 est le principal senseur du toucher [12] et de la proprioception [13], le sens de la position du corps et de ses mouvements. II ne joue qu'un rôle marginal dans la détection de la douleur mécanique, dont les senseurs, qui sont inconnus à ce jour, font l'objet de recherches intensives.

La découverte des canaux ioniques mécanosensibles Piezo a permis de comprendre les mécanismes fondamentaux de la perception du toucher, mais leurs fonctions ne se résument pas à la somesthésie. En effet, toutes les cellules de notre organisme sont soumises à des contraintes mécaniques, et ces senseurs sont exprimés dans de multiples tissus. L'étude de souris déficientes pour ces gènes a montré leur implication dans de nombreuses fonctions physiologiques et développementales, telles que la mise en place des systèmes vasculaire [14] et lymphatique [15], et l'homéostasie des globules rouges [16]. Chez l'homme, les tableaux cliniques multi-symptomatiques qui accompagnent certaines mutations des gènes PIEZO1 ou PIEZO2 illustrent d'ailleurs le rôle qu'ils jouent dans de nombreux processus biologiques.

La recherche sur les mécanismes moléculaires et cellulaires qui gouvernent nos sens a toujours bénéficié d'une attention particulière du comité du prix Nobel. Ce fut le cas de l'audition en 1961 (Georg von Békésy), de 
la vision en 1967 (Ragnar Granit, Haldan Keffer Hartline et George Wald) et de l'odorat en 2004 (Richard Axel et Linda Buck). Le prix Nobel de physiologie ou médecine 2021 s'inscrit dans cette dynamique, en mettant à l'honneur la découverte des canaux ioniques activés par la température et les forces mécaniques, qui constituent les senseurs moléculaires de la somesthésie.

The Nobel Prize in Physiology or Medicine 2021:

Temperature and touch sensors

\section{LIENS D’INTÉR $\hat{T}$}

L'auteur déclare n'avoir aucun lien d'intérêt concernant les données publiées dans cet article.

\section{RÉFÉRENCES}

1. Caterina MJ, Schumacher MA, Tominaga M, et al. The capsaicin receptor: a heat-activated ion channel in the pain pathway. Nature 1997 ; 389 : 816-24.

2. Caterina MJ, Leffler A, Malmberg AB, et al. Impaired nociception and pain sensation in mice lacking the capsaicin receptor. Science $2000 ; 288$ : 30613.

3. Tominaga M, Caterina MJ, Malmberg AB, et al. The cloned capsaicin receptor integrates multiple pain-producing stimuli. Neuron $1998 ; 21: 531-43$.

4. McKemy DD, Neuhausser WM, Julius D. Identification of a cold receptor reveals a general role for TRP channels in thermosensation. Nature 2002 ; $416: 52-8$.
5. Peier AM, Mogrich A, Hergarden AC, et al. A TRP channel that senses cold stimuli and menthol. Cell $2002 ; 108: 705-15$.

6. Dhaka A, Murray AN, Mathur J, et al. TRPM8 is required for cold sensation in mice. Neuron 2007 ; $54: 371-8$.

7. Story GM, Peier AM, Reeve AJ, et al. ANKTMI, a TRP-like channel expressed in nociceptive neurons, is activated by cold temperatures. Cell $2003 ; 112: 819-29$.

8. Macpherson LJ, Dubin AE, Evans MJ, et al. Noxious compounds activate TRPAl ion channels through covalent modification of cysteines. Nature $2007 ; 445: 541-5$.

9. Koivisto AP, Belvisi MG, Gaudet R, Szallasi A. Advances in TRP channel drug discovery: from target validation to clinical studies. Nat Rev Drug Discov 2021. https://doi.org/10.1038/s41573-02100268-4.

10. Coste B, Mathur J, Schmidt M, et al. Piezol and Piezo2 are essential components of distinct mechanically activated cation channels. Science $2010 ; 330: 55-60$.

11. Coste B, Xiao B, Santos JS, et al. Piezo proteins are pore-forming subunits of mechanically activated channels. Nature 2012 ; 483 : 176-81.

12. Ranade SS, Woo SH, Dubin AE, et al. Piezo2 is the major transducer of mechanical forces for touch sensation in mice. Nature $2014 ; 516: 121-5$

13. Woo SH, Lukacs V, de Nooij JC, et al. Piezo2 is the principal mechanotransduction channel for proprioception. Nat Neurosci 2015 ; 18 : 1756-62

14. Ranade SS, Qiu Z, Woo SH, et al. Piezol, a mechanically activated ion channel, is required for vascular development in mice. Proc Natl Acad Sci USA 2014 ; 111 : 10347-52.

15. Nonomura K, Lukacs V, Sweet DT, et al. Mechanically activated ion channel PIEZOl is required for lymphatic valve formation. Proc Natl Acad Sci USA 2018 ; 115 : 12817-22.

16. Cahalan SM, Lukacs V, Ranade SS, et al. Piezol links mechanical forces to red blood cell volume. Elife 2015 ; 4.

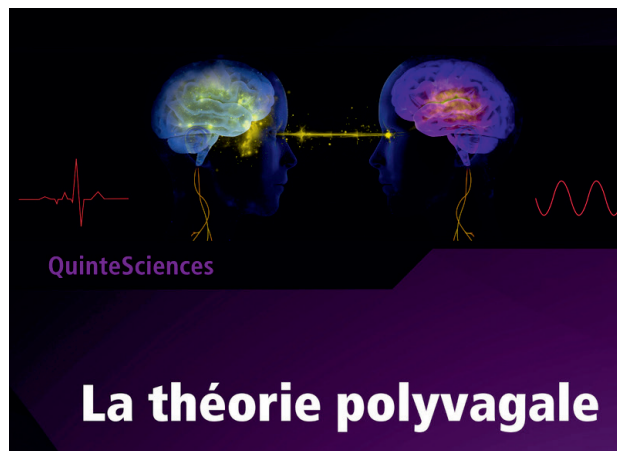

Fondements neurophysiologiques des émotions, de l'attachement, de la communication et de l'autorégulation

Stephen W. Porges

Traduit par Nico Milantoni et Isabelle Chosson-Argentier

\section{edpsciences}

\section{Enfin disponible en français}

\section{L'ouvrage de référence de S.W.Porges}

S.W.Porges est le spécialiste mondial du lien unissant le système nerveux autonome au comportement social. Il nous offre de passionnantes perspectives sur la façon dont notre système nerveux autonome gère inconsciemment notre engagement social, la confiance, l'intimité.

La traduction a été réalisée par des experts : Nico Milantoni est psychologue, praticien et formateur de la méthode Hipérion. Isabelle Chosson-Argentier est Docteur en pharmacie, conseil en nutrition, micronutritionniste, phyto- et aromathérapeute, praticienne de la méthode Hipérion.

\section{3 pages $-69 €$ TTC}

ISBN : 978-2-7598-2498-4

En vente sur la boutique.edpsciences.org 